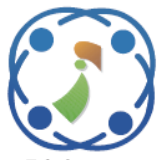

\title{
Moving Target Tracking and Classification Based on Micro-Doppler Signatures
}

\author{
Thamir R. Saeed ${ }^{1 *}$ \\ Mahmuod Hamza Al-Muifraje ${ }^{1}$ \\ Jafar W. Abdul Sadah' \\ ${ }^{1}$ Department of Electrical Engineering, University of Technology, Iraq \\ ${ }^{2}$ Department of Communications Engineering, University of Baghdad, Iraq \\ ${ }^{3}$ Faculty of Medicine, University of Iraq, Iraq \\ * Corresponding author's Email: thamir_rashed@yahoo.com
}

Aqeela N. Abed ${ }^{3}$

\begin{abstract}
This paper presents the results of accompanying classification of tracking for different classes of targets such as a car (moving non-rigid target), moving people (slow moving non-rigid target). The Data are collected using frequency modulation continuous wave (FMCW)radar, while different neural algorithms are considered for classification of targets recorded. A Doppler and Micro Doppler have been used as target features, while short time Fourier transform (STFT) has been used as a feature extraction algorithm. A novel combination between Kalman filter and tree-structured, as well as self-organizing map (SOM) neural network has been proposed as an estimator and classifier, respectively. The results show that the proposed approach is overperformed to the conventional classifier SVM by $1.6 \%$ and the gained classification is $92.6 \%$, while the reduction error due to using Kalman filter is $95 \%$.
\end{abstract}

Keywords: Moving target tracking, Micro-doppler, Moving target classification.

\section{Introduction}

In surveillance systems, video cameras represent the most used sensors. Those cameras give a highly elaborated description of a condition that is smoothly interpreted by both humans and computers. However, two factors are influencing this description; when being recorded in less than ideal circumstances and at long distance. Therefore, a radar sensor is a powerful solution for these situations [1]. The Radar sensors provide the advantage of not being affected by light and weather conditions, as well as exploiting mature rangeDoppler [2]. The Doppler-range is the change in the transmitted frequency caused by range variation (object motion). However, moving the smaller parts of that object can cause an additional Doppler (micro-Doppler) which is at the sideband of the Doppler frequency of the moving object. The microDoppler map can be seen as the power reflected as a function of the speed of the reflector $[1,3]$. Therefore, the Doppler and micro-Doppler have been used in classification processing for different end applications over the years [2]. However, the applicability and adaptation of the Doppler and Micro-Doppler frequency techniques to the specific application context, and the development of the most suitable processing to fuse information from different radar channels and heterogeneous sensors are still open research questions. [4].

In this context, one of the most important applications is the classification and tracking of targets in urban areas because they are full with different objectives to be observed. In such environments, a wide variety of moving objects such as Humans, vehicles, birds, and animals may lead to a large number of closely spaced radar detections that can cause severe problems in the tracking process such as false tracks, merge tracks, and track loss. By extracting non-kinematic features such as micro-Doppler signatures in 1-D or 2-D from the radar signal, the radar detections can be classified in different target classes to mitigate the problems in the tracking process and improve situation awareness[5]. 
The micro-Doppler properties of a target are determined by variation or translating or rotating parts in addition to the bulk target motion. The number, location, and the type of motion of such parts are specific for a given target type. Consequently, the related micro-Doppler signature can be exploited for classification [6,7]. Analysis of the micro-Doppler signature may provide specific properties of rotating parts such as rotation rate. However, reliable target classification using microDoppler signature analysis imposes severe requirements on the radar waveform [5].

The detection, tracking and then the classification of moving targets are of interest in literature micro-Doppler signatures had been used in $[7,8]$ to discriminate and to track the small UAV using the multi-static radar system. [3] presents the investigation of the human motion action under four different environments. The feature was extracted by using STFT, while seven features were used to classify that human action based on SVM and RBF. The drop of his classification is caused by two reasons, the first one is not using the optimal features, while the other reason is the increase in standard deviation caused by environmental noise. Therefore, the classification rate was gained is 80$90 \%$. While the drawback of [9] is the use of standard constant false alarm rate (CFAR) and track the target without prior information about the angle. In [5] the range-Doppler was used to classify the air target among other targets which is a traditional classification and he did not use the micro-Doppler for a specific and accurate classification. [10] presented a deep conventional neural network (DCNNs) for human detection and activity classification based on Doppler radar. The results shown that the DCNN could achieve accurate results of $97.6 \%$ for human detection and $90.9 \%$ for human activity classification. On the other hand, the limitations of the work can be summarized by the following; did not use any explicit domain knowledge for extracting features, and the performance can degrade if there exist variations due to the irregularity in the object motions, and the complexity of DCNNs is usually higher than that of data-driven models from regular machine learning algorithms. [1] investigated the performance of structured, which is used for personal identification. He used a camera, radar, and the error rates ranging from $0 \%$ to $6.5 \%$ for actions recognition and $10 \%$ to $12 \%$ for person identification. Consequently, the error of his classification rate was due to the classification model depended on the number of trained epochs of the SIN only and without consideration of the targets.
[11] presented the recognition of dynamic hand gestures using two radar sensors with angular diversity. The recognition accuracy was related to the two radar sensors orthogonality to each other. The results showed that the classification of one radar using SVM as a classifier is $87 \%$, while for two radars with the same classifier is $92 \%$. It seems that the noise is not in the account of the radar processing which is leading to reduce the classification. [9] presented an ultra-wideband shortrange radar (UWB-SRR) recognition system based on higher order statistics (HOS) and support vector machines (SVMs) and combined with CA-CFAR for road safety. The results showed that the recognition rates were $92.77 \%$ of the cyclist, $82 \%$ of the pedestrian and $90.73 \%$ of the car. The classification rate reduced due to using CA-CFAR, which is working with the homogenous environment and his work is not.

Although many researchers presented many topics of the classification of moving target based on the Doppler and micro-Doppler, a little attention has been paid to the continuation of that classification with variable acceleration's moving targets.

However, the detection and tracking based on the micro-Doppler features represent a robust technique, the convergence of Kinetic dynamics of some target leads to the convergence of their MicroDoppler (MD) between them, hence the Kalman filter (KF) can be utilized to estimate the precision Doppler which would result in higher accuracy to detect and classify, consequently to track these targets. Therefore, the use of the Kalman filter assists the system to become a more accurate technique. Where the Kalman filter [12] has been represented as a fast recursive linear filter, it used to estimate the dynamic system of noisy measurements. Kalman filters can estimate the internal state of model variables that cannot be measured directly. Also, it is based on the recursive structure, low computational demand, and robust against measurement inaccuracies and correlations in a multi-target scenario $[13,14,15]$. Also, one benefits of the Kalman filter is the prediction of the next value, which is useful if the loss of the target for a certain period of time.

So this paper presents an accompanying classification of tracking for different classes of targets based on a tracking filter and efficient classifier. After extracting the Doppler features using short time Fourier transform (STFT), the Kalman filter is used to estimate the Doppler and micro-Doppler of a moving target. Then a treestructured, self-organizing map (SOM) neural network is used as the classifier. The combination of 
estimated filter and the classifier formed a novel strategy, which significantly increases the classification rate.

Therefore, the proposed work can be considered as the treatment of the two important drawbacks of the previous work; the low classification rate caused by the environmental noise and thus the classification will be affected and gets an error on it.

Therefore, the combination of using the Kalman Filter with the tree-structured, self-organizing map (SOM) neural network can satisfy this treatment. Where, the Kalman filter estimates the measured Doppler values which can reduce the noise of the environment and target motion, while the neural network classify the estimated measurements.

According to this, the paper organized as, section 2 is the tracking model, while section 3 is the classifier model. Section 4 is the explanation of the proposed system, while the results and discussion are presented in section 5 and finally the conclusion in section 6 .

\section{The tracking model}

The state of moving targets at time $k$ is a twodimensional vector; position $X_{k}^{p}$ and speed $X_{k}^{p^{\prime}}$ components [8,16];

$$
X_{k}=\left(X_{k}^{p}, X_{k}^{p^{\prime \prime}}\right)
$$

While the position and speed components of the targets are;

$$
X_{k}^{p}=(x, y), \quad X_{k}^{p^{\prime}}=\left(x^{\prime}, y^{\prime}\right)
$$

The radar measurement components are;

$$
r=h\left(X_{k}\right)+w
$$

Where;

$h\left(X_{k}\right)$ - Measurement function of the target components $\left(X_{k}\right)$, which is depends on the receiver parameters such as antenna and low noise amplifier.

$w$ - measurement noise.

The tracker (Kalman) filter estimates the target state and the position components as;

$$
\hat{X}_{k}=\left(\hat{x}, \hat{y}, \hat{x}^{\prime}, \hat{y}^{\prime}\right) \quad \text { and } \quad \hat{X}_{k}^{p}=(\hat{x}, \hat{y})
$$

The target motion model is;

$$
X_{k+1}=\mathbf{F} \cdot X_{k}+\mathrm{V}
$$

Where;

$\mathrm{V}$ - process noise with covariance $\mathrm{Q}$ and acceleration's variances $=10 \frac{\mathrm{m}}{\mathrm{s}^{2}}$.

F - transition matrix.

The target position stat prediction is;

$$
\widehat{X}_{k+1}=\mathbf{F} \cdot \hat{X}_{k}
$$

The predicted error covariance matrix is [17];

$$
\mathbf{P}_{k+1 / k}=\mathbf{F P}_{k / k} \mathbf{F}^{T}
$$

The Kalman filter has estimated the radar measurements as;

$$
\hat{X}_{k+1 / k+1}=\hat{X}_{k+1 / k}+\mathbf{K} \cdot\left(r \cdot h\left(X_{k+1}\right)\right)
$$

The Kalman weight is;

$$
\mathbf{K}=\mathbf{P}_{k+1} \mathbf{H}_{k+1}^{T} \mathfrak{C}^{-1}
$$

Where;

$\mathfrak{C}-$ innovation matrix $=\mathbf{H}_{k+1} \mathbf{P}_{k+1 / k} \mathbf{H}_{k+1}^{T}+R$

R- observation covariance matrix noise

Then, the estimated error covariance matrix is;

$$
\mathbf{P}_{k+1 / k+1}=\left(I-\mathbf{K H}_{k+1}\right) \cdot \mathbf{P}_{k+1 / k}
$$

In this work, the estimation and prediction are carried out to the Doppler and the rate of the Doppler (the phase). Therefore $X_{k}^{p}$ will be represented a Doppler vector, while $X_{k}^{p^{\prime}}$ represents the rate of that Doppler (phase vector). Then, state space components are;

$$
X_{k}=\left(X_{k}^{p}, X_{k}^{p^{\prime \prime}}\right)
$$

\section{The classifier model}

Tree-structured, hybrid neural network and SOM are based on multi-layers, each layer has some subnet SOM. Where the input feature vector with low resolution for the unknown target is fed to the input layer, then the output of the first layer is more resolution by obtaining the winner node, which is fed to the second layer. Then, this algorithm passes on a layer-by-layer until the determination of the target class label. 
In this context, the SOM network represents the unsupervised learning method, where, it is weighted adaptively by self-organizing competition. Where the output is represented winner if it satisfies the minimum Euclidean distance between model vector $\boldsymbol{W}_{c}$ ( index c (best match)), and $\mathbf{X}$ (input vector)as follows [18];

$\left\|\boldsymbol{X}-\boldsymbol{W}_{c}\right\|=\min _{j}\left\{\left\|\boldsymbol{X}-\boldsymbol{W}_{j}\right\|\right\} \quad(j=1,2, \ldots \ldots, K)$

Where;

$$
\begin{gathered}
\boldsymbol{X}=\text { input sample vector }=\left[\boldsymbol{x}_{1}, \boldsymbol{x}_{2}, \ldots \ldots \boldsymbol{x}_{N}\right]^{\boldsymbol{T}} \\
\boldsymbol{W}_{j}=\text { weight vector of } j \text { th neuron }= \\
\quad\left[W_{j 1}, W_{j 2}, \ldots . W_{j N}\right]^{T}(\mathrm{j}=1,2, \ldots \mathrm{K})
\end{gathered}
$$

The weight vectors are updated using a weight adaptation function based on the following rule [19];

$$
\left\{\begin{array}{c}
\boldsymbol{W}_{i}(t+1)=\boldsymbol{W}_{i}(t)+\alpha(t)\left[\boldsymbol{X}(t)-\boldsymbol{W}_{i}(t)\right] \\
i \in N_{c}(t) \\
\boldsymbol{W}_{i}(t+1)=\boldsymbol{W}_{\boldsymbol{i}}(t) \\
i \notin N_{c}(t)
\end{array}\right\}
$$

Where;

$$
\begin{aligned}
& \alpha(t) \\
& - \text { learning rate decreases monotonically with } \\
& \quad \text { time } t \text {. }
\end{aligned}
$$$$
N_{c}(t) \text { - topological neighborhood function }
$$
decreases monotonically with time $t$.

The radar parameters which are used to illuminate the moving target are illustrated in Table 1 [20,21].

In this work, the vector $\boldsymbol{X}$ represents the estimated vector of the Eq. (10) according to the algorithm from the Eqs. (1) to (9).

Table 1. Experimental and simulation parameters

\begin{tabular}{|c|c|c|}
\hline No. & Parameters & Quantity \\
\hline 1 & Waveforms & FMCW/FSK/CW \\
\hline 2 & Carrier Frequency & $2.4 \mathrm{GHz}$ \\
\hline 3 & BW & $440 \mathrm{MHz}$ \\
\hline 4 & Sampling & 128 \\
\hline 5 & $\begin{array}{c}\text { Sweep Time } \\
\text { FMCW-Sawtooth }\end{array}$ & $0.5 \mathrm{msec}$. \\
\hline 6 & Ant. Polarization & $\mathrm{V}\left(38^{\circ}\right)$ \\
\hline 7 & Noise Figure & $2.8 \mathrm{~dB}$ \\
\hline 8 & Power output & $22 \mathrm{dBm}$ \\
\hline
\end{tabular}

\section{The proposed system}

To improve the detection and tracking system based on micro-Doppler frequencies, a treestructured, SOM neural network and has been used after Kalman filter as shown in Fig. 1.

All data have been collected using the AncortekSDR-KIT 240B [20] fully integrated automotive radar transceiver manufactured by Ancortek. The radar operation mode was configured as a frequency modulated continuous wave (FMCW), with linear chirp modulation, and the parameters, shown in Table 1 . These parameters were empirically found to provide the clearest MD signatures at visual inspection, as well as providing a reasonable compromise in terms of range resolution, Doppler unambiguous range, and data throughput for fast transferring and processing. The system had one transmitter and one receiver channels, and digitized data were transferred from the Kit unit to a computer.

The Data are analyzed using STFT in which the Doppler and micro-Doppler are extracted as features. After that, such features will be estimated and predicted by the Kalman filter to reduce the noise and predict the next value. Then they will be classified by using a multilayer SOM classifier.

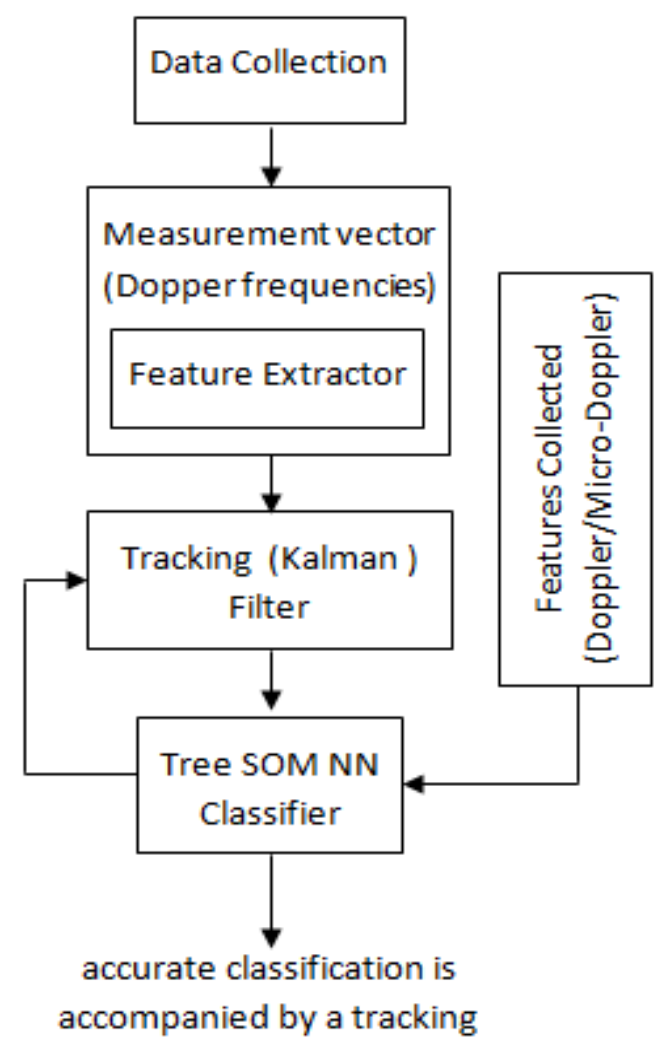

Figure. 1 Proposed system block diagram 


\section{Results and discussion}

The results obtained in previous studies $[1,3,9$, 11] using SVM as a classifier indicated that the classification rate is $87-92 \%$. According to that, the result of [3] is considered acceptable, while the result of the [11] can be considered as good results. Although, the [11] uses two radar, therefore this result is considered expensive. In this context, in [9] uses the CA-CFAR with high order statistics with 1000 signatures, which are led to the complexity of the classification process. While [1] is use camera with the radar, I agree with the author for safety in the road, while it is considered more expensive.

In spite of these works are classified the target, but not tracked it, i.e the performance are not continually.

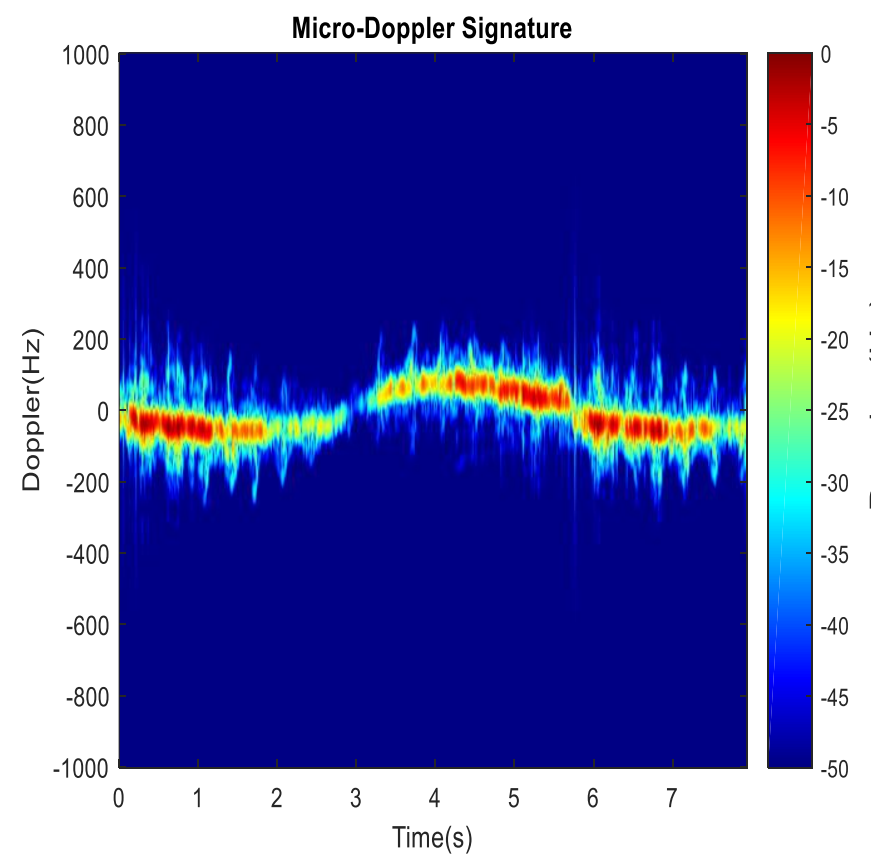

(a)

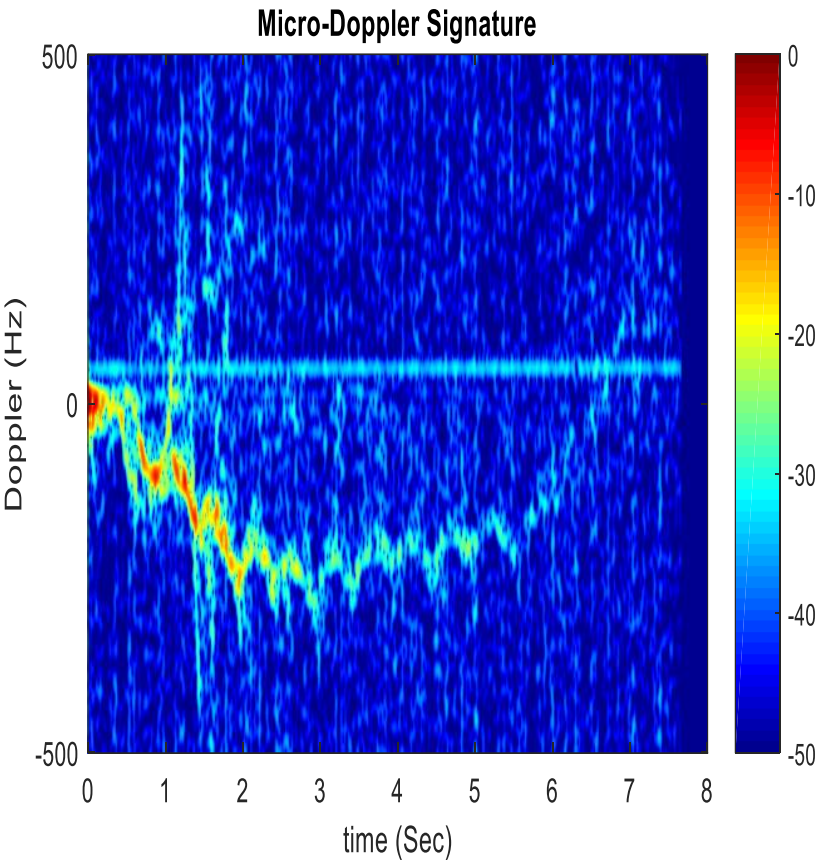

(b)

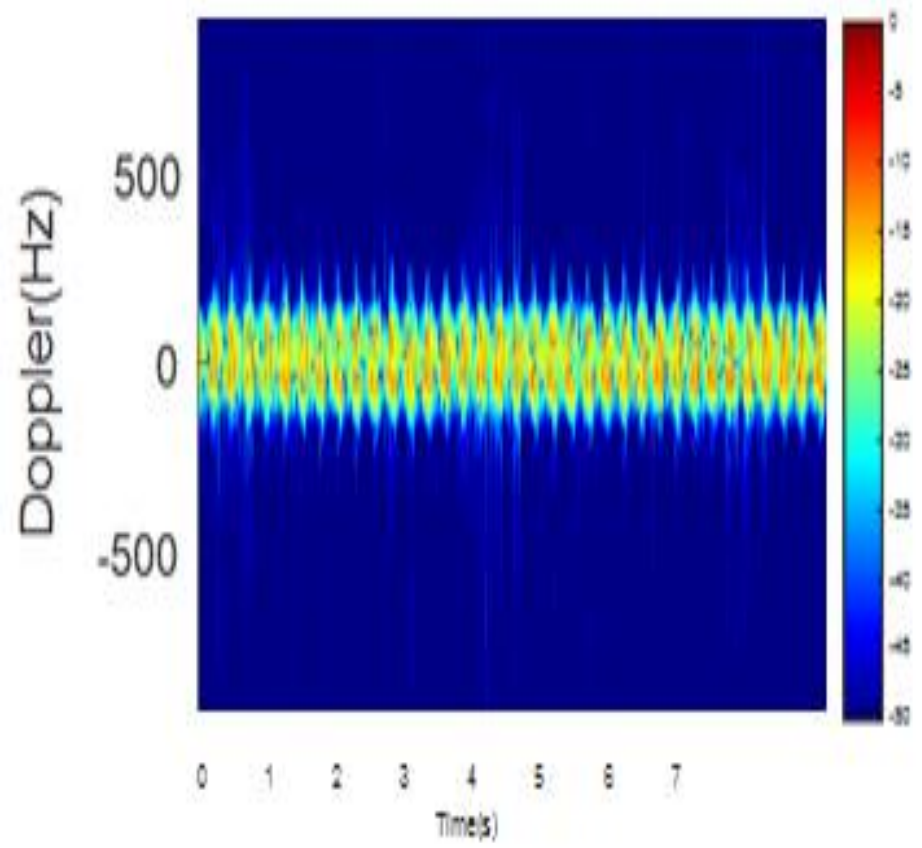

(c)

Figure. 2 Targets doppler and micro-Doppler of two moving targets: (a) Target A 0-155 m/sec, (b) Target B 0-66 m/sec, and (c) Target C (0-5.5) $\mathrm{m} / \mathrm{sec}$ 
In this study, three targets have been tracked at different speeds $(0-150 \mathrm{~m} / \mathrm{Sec}$.) and with a different motion of interior parts, of those targets. Target (A), which represent a car with different speed and acceleration (small cars). Where, the speed of this target type is $0-155 \mathrm{~m} / \mathrm{sec}$ as the maximum. The normal speed of this type is $(16.66-55.55 \mathrm{~m} / \mathrm{sec}$.) in the urban environment with acceleration (0-22.22 $\mathrm{m} / \mathrm{sec}$. will be reach during nearly 10-20 Sec.). Target (B) is a car with the same normal (not the maximum speed) speed of target (A), but with low acceleration (0-22.22 m/sec. will reach during nearly 20-30 Sec.) (buses and school buses). On the other hand, target $(\mathrm{C})$ is a walking person, the speed of this type is $(1.666-5.55 \mathrm{~m} / \mathrm{sec}$.). Note that, all these targets have interior moving parts, and all those targets are conflicted in urban environments. The analysis of these targets is shown in Fig. 2, which represents the extracted Doppler and micro-Doppler of these targets, and the micro-Doppler of its interior parts.

Fig. 2 (a) shows the Doppler of target A caused by its motion. It is clear from this figure there are a Doppler value (black red color) of the main object (target) motion which is main Doppler value, while the values around that main Doppler were the microDoppler of the object's parts. The micro-Doppler values are indicated by other color around the black red. Where the color depends on the Doppler and Micro-Dppler values. Figs. 2 (b) and (c) show the Doppler and Micro-Doppler of the targets B and C as in target $\mathrm{A}$, the clear difference between these targets is by the acceleration. Where, the target $\mathrm{A}$ has an acceleration, greater than the acceleration of $\mathrm{B}$ and $\mathrm{C}$, and the acceleration of $\mathrm{B}$ is greater than that of $\mathrm{C}$. In this context, the Micro-Doppler which is related to targets parts, where the Micro-Doppler of the target $\mathrm{A}$ is greater than the other targets and the micro-Doppler of the target B is greater than the micro-Doppler of the target $\mathrm{C}$.

It should be noted the effect of the noise and the change of the velocities on Doppler and microDoppler of the mentioned targets is shown in Figs. 2 (a) and (b) especially.

There is obviously a companion noise to the Doppler extraction, Where the source of that noise is coming from the surrounding closest non-rigid moving targets which have non-constant velocities. Therefore, Fig. 3 appears the redaction of that noise as a result of using Kalman filter. Where, the power and the accuracy of the operation of Kalman filter depending on the determination of the possibly moving targets in the environments. Then, the Doppler and micro-Doppler of these targets is determined, therefore, the reduction of its effect will be done. After that, the accuracy of determining the Doppler and micro-Doppler of the desired target will be gained.

Therefore, the unwanted targets in the field of operation like birds are determined and then reduce its effects on the measurements of wanting targets by using $\mathrm{KF}$.

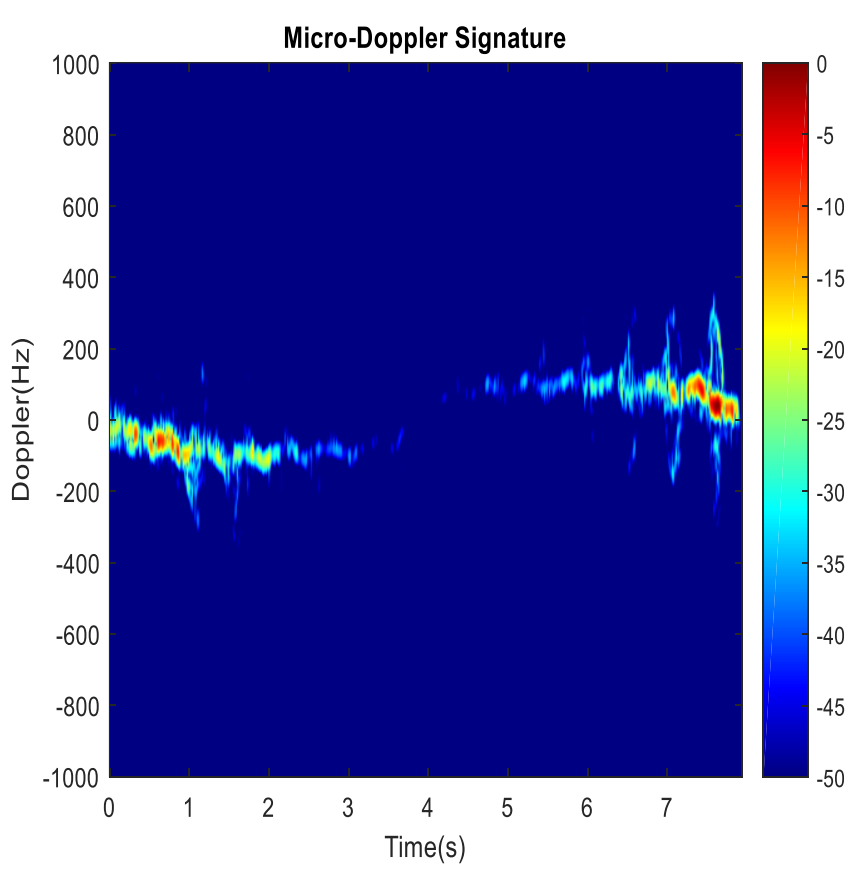

(a)

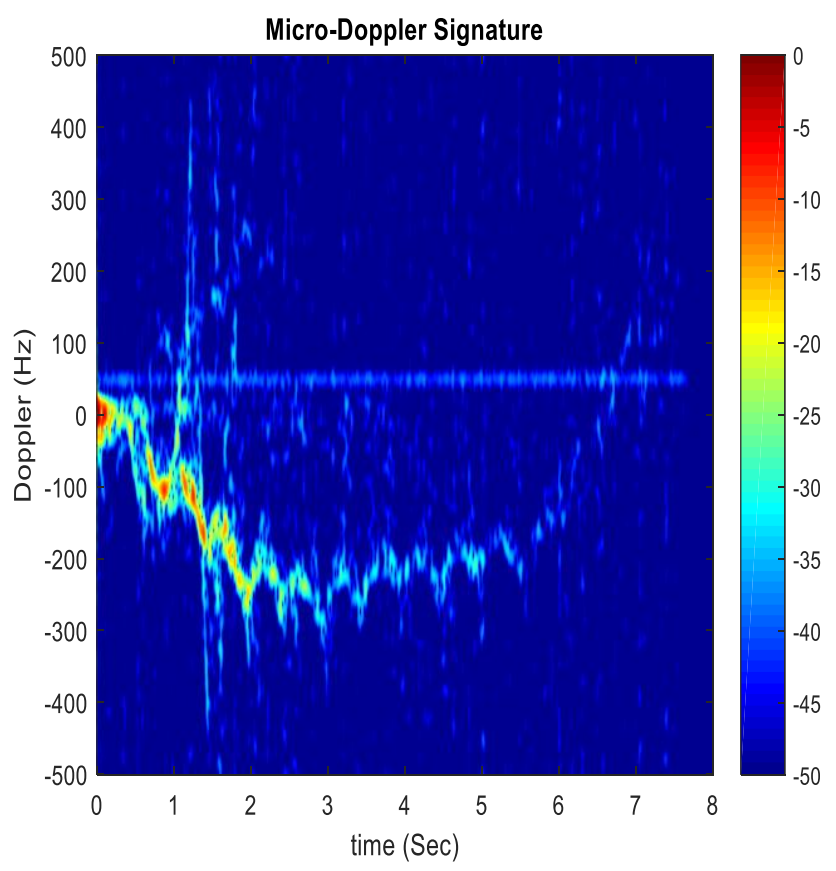

(b)

Figure. 3 Targets doppler and micro-doppler of two moving targets after Kalman filter: (a) Target A 0-155 $\mathrm{m} / \mathrm{sec}$ and (b) Target B 0-66 m/sec 
In this context, the walking person (target $\mathrm{C}$ ) has a little noise compared with that of the car (targets A and B), therefore the results of the target (C) by using Kalman filter has not appeared here.

For more illustration, Fig. 4 shown the simulation of the measurements, estimated and the truth values of the Doppler of the tracking targets. It is clear the reduction of the error which is caused by the interfered Doppler of the unwanted moving targets.

On the other hand, it can be observed that during the sharp change of the velocity, the error is increased as indicated in Fig. 4. Also, the effect of the Kalman filter to reduce that error is shown in that figure. Where, the KF reduction is based on the prior knowledge of the wanted and unwanted targets.

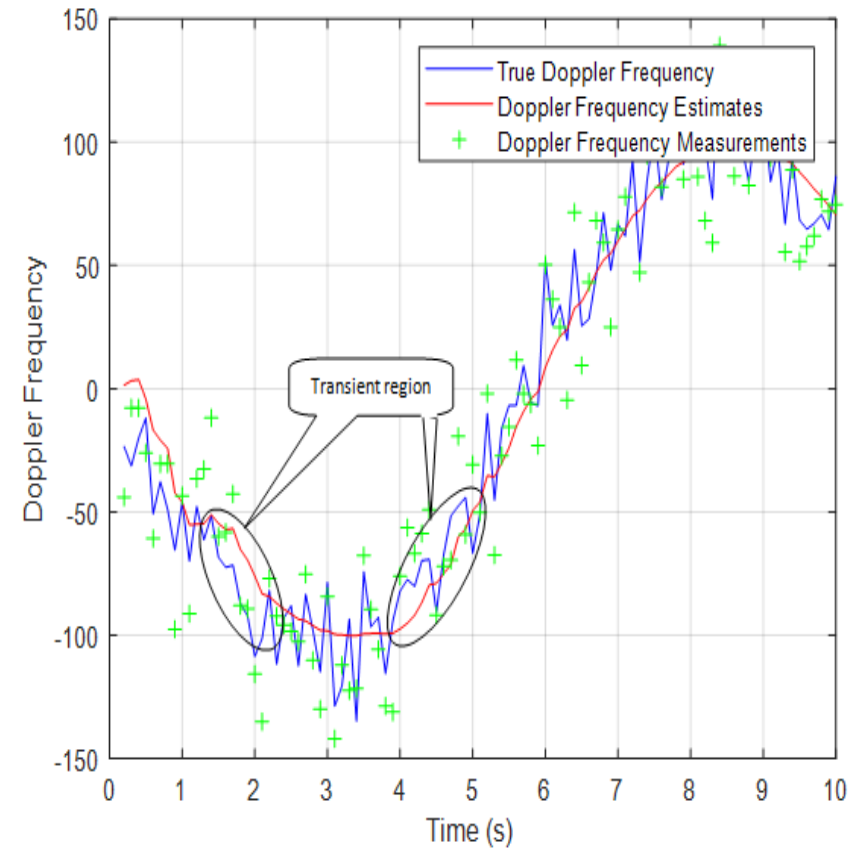

(a)

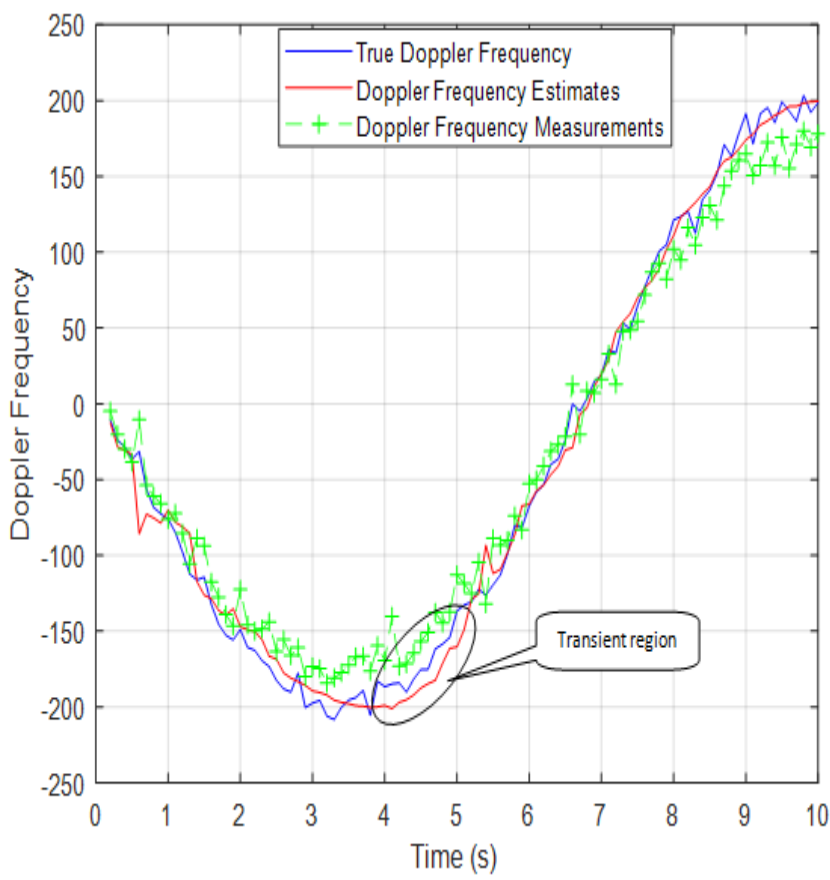

(b)

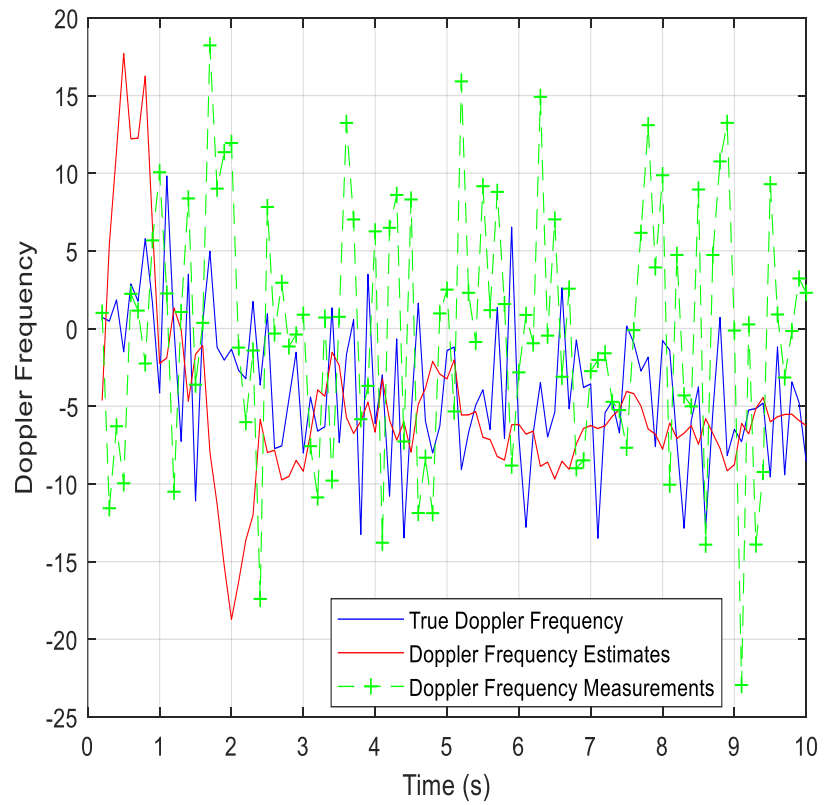

(c)

Figure. 4 The effects of target velocity variation and Kalman filter: (a) doppler frequency of Target A 0-155 m/sec, (b) doppler frequency of Target B 0-66 m/sec, and (c) Target C (0-13.8) $\mathrm{m} / \mathrm{sec}$ 


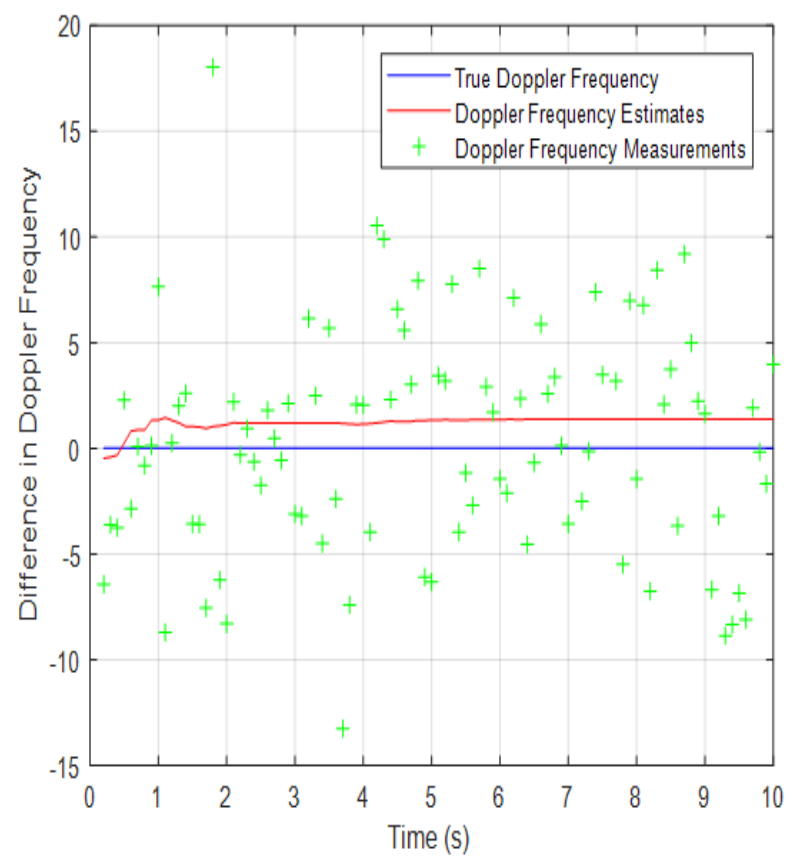

(a)

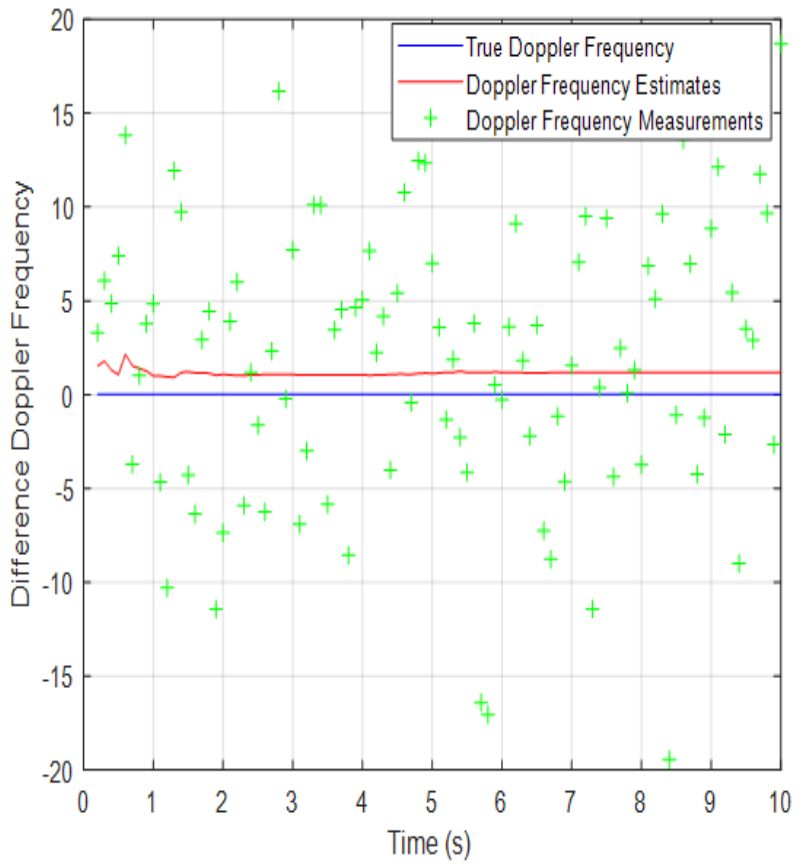

(b)

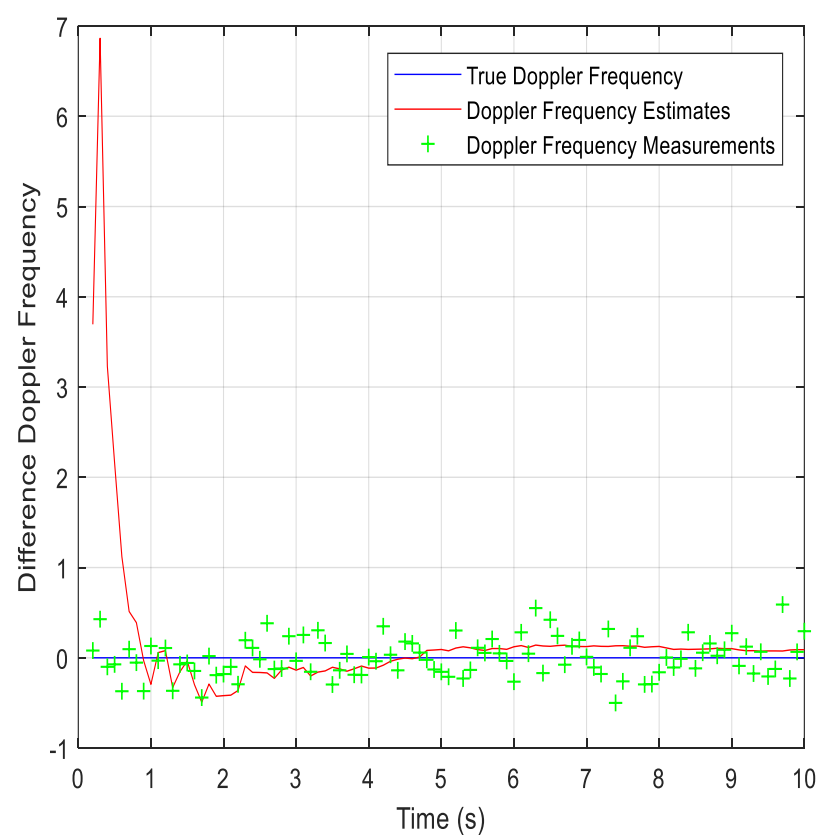

(c)

Figure. 5 Average error with and without the Kalman filter of doppler frequency (DF) measurements: (a) the difference of DF of TGT A 0-155 m/sec, (b) the difference of DF of TGT B 0-66 m/sec, and (c) the difference of DF of TGT A 0$13.8 \mathrm{~m} / \mathrm{sec}$

Quantitative analysis to determine the average error was applied, based on the maximum deviation of the measured Doppler and micro-Doppler according to the velocity variations of the moving targets within the considered period. The average error of the Doppler frequency (DF) measurements, in the measurement period, and the effect of the Kalman estimator with real data are shown in Fig. 5.
It can be observed that using of the Kalman filter estimator has improved the Doppler feature extraction by reducing the error about $94 \%$. It should be noted that, the mean square error of the measurement before and after Kalman filter stage has been evaluated as in Table 2 . 
Table 2. Mean square error with and without Kalman

\begin{tabular}{|c|c|c|c|}
\cline { 2 - 4 } \multicolumn{1}{c|}{} & $\begin{array}{l}\text { MSE for } \\
\text { Measurement }\end{array}$ & $\begin{array}{l}\text { MSE for } \\
\text { Estimated }\end{array}$ & $\begin{array}{l}\text { KF } \\
\text { Improvement }\end{array}$ \\
\hline $\begin{array}{c}\text { Target } \\
\text { A }\end{array}$ & 0.0102 & $6.865 \times 10^{-4}$ & $93 \%$ \\
\hline $\begin{array}{c}\text { Target } \\
\text { B }\end{array}$ & 0.025 & 0.0014 & $94 \%$ \\
\hline $\begin{array}{c}\text { Target } \\
\text { C }\end{array}$ & 0.001 & 0.0005 & $99 \%$ \\
\hline
\end{tabular}

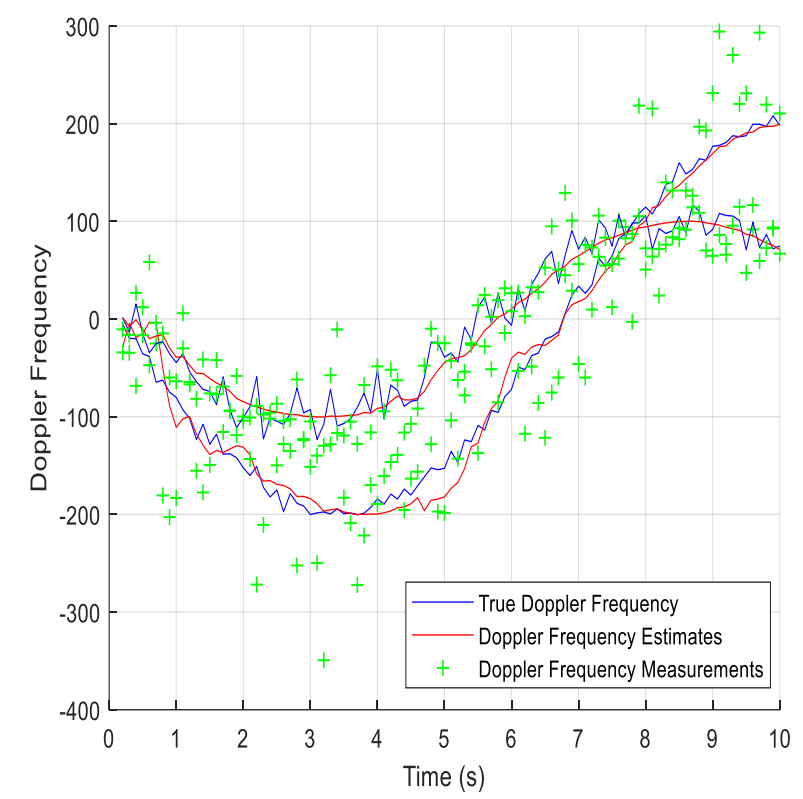

Figure. 6 Two dimensional Kalman filter for two targets
After that, two dimensional Kalman filter has been designed for tracking the two targets as shown in Fig. 6. It can be noted that the error is slightly increased when the target velocity is varied sharply. It is clear that using the Kalman filter was the main reason for minimizing the error and making it slightly.

For the comparison with the other works, we had to apply some of these work as mentioned in the sources, where [3] he used SVM and SOM as classifiers. After that, we applied our proposal, which requires the addition of Kalman filter candidate before SOM for improving the accompanying classification of tracking, which is applied with different targets. Also, for the refs [1, 9, 11], where they used the SVM as a classifier, while for ref. [11], we applied our proposal with one radar, which is representing one part of this work.

In this context, it is evident that the classification results obtained here for SVM and SOM or only SVM without Kalman filter are in exceptional agreement with those reported in $[1,3,9,11]$ results in free space. While the results demonstrate that the improved in classification was achieved by using a Kalman filter as shown in Table 3, In addition to, the continuous classification has been gained.

Table 3. Comparison of the present work with previous work

\begin{tabular}{|c|c|c|c|c|c|c|c|}
\hline & & & & \multirow{4}{*}{$\begin{array}{l}\text { Classification } \\
\text { Algorithm }\end{array}$} & \\
\hline & & & \multicolumn{3}{|c|}{ Classification Rate $\%$} & & \multirow{3}{*}{$\begin{array}{c}\text { Average } \\
\text { Classification } \\
\%\end{array}$} \\
\hline & & & & Features & & & \\
\hline \multicolumn{3}{|c|}{ Works } & $\begin{array}{l}\text { 1-Average } \\
\text { Doppler } \\
\text { frequency } \\
\text { 2- Total } \\
\text { bandwidth }\end{array}$ & $\begin{array}{l}\text { 1- Doppler offset } \\
\text { 2- Bandwidth } \\
\text { without MDS } \\
\text { 3- Total bandwidth }\end{array}$ & $\begin{array}{l}\text { 1-Average } \\
\text { Doppler } \\
\text { frequency } \\
\text { 2-Total bandwidth } \\
\text { 3-Doppler offset } \\
\text { 4-Bandwidth } \\
\text { without MDS*. }\end{array}$ & & \\
\hline \multicolumn{3}{|c|}{1} & \multicolumn{3}{|c|}{ The feature in this work is Micro-Doppler } & SVM & 90 \\
\hline \multicolumn{3}{|c|}{3} & 86 & 89.44 & 98.11 & SVM & 91.1 \\
\hline \multicolumn{3}{|c|}{9} & \multicolumn{3}{|c|}{ The feature in this work is High order statistics } & SVM & 91.44 \\
\hline \multirow[t]{3}{*}{11} & \multirow[t]{2}{*}{$\begin{array}{l}\text { One } \\
\text { Radar }\end{array}$} & $\begin{array}{l}\text { Without } \\
\text { KF }\end{array}$ & \multirow{3}{*}{\multicolumn{3}{|c|}{$\begin{array}{l}\text { The feature in this work is Time, Frequency and } \\
\text { estimated position }\end{array}$}} & SVM & 87 \\
\hline & & With KF & & & & & 91 \\
\hline & \multicolumn{2}{|c|}{ Two Radar } & & & & SVM & 92 \\
\hline \multirow{4}{*}{$\begin{array}{l}\text { Present } \\
\text { work }\end{array}$} & \multicolumn{2}{|c|}{ Without KF } & 85 & 90 & 98.5 & \multirow[b]{2}{*}{ SVM } & 91.1 \\
\hline & \multicolumn{2}{|c|}{ With KF } & 88.11 & 90 & 99 & & 92.3 \\
\hline & \multicolumn{2}{|c|}{ Without KF } & 84 & 87.4 & 98.12 & Tree- & 89.6 \\
\hline & \multicolumn{2}{|c|}{ With KF } & 88.2 & 91 & 99.1 & $\begin{array}{l}\text { structured, } \\
\text { self- } \\
\text { organizing } \\
\text { map (SOM) } \\
\text { neural } \\
\text { network } \\
\end{array}$ & 92.6 \\
\hline
\end{tabular}




\section{Conclusion}

Many applications need continuous monitoring of objectives (moving targets) within the scope of application, particularly if these objectives are possible to conflicting and cross. One of the most important applications is the bustling urban environments. There are many types of targets in these environments; a proposal has been presented The overperform of the proposed work is clear, where it is advance the Refs $[1,3,9,11]$ by $1.2-6 \%$. This advancement is a result of using KF and SVM with SOM as a classifier. In the other hand, the drawback of this work is increasing of the complexity caused by adding the KF, while this defect can be overlooked by increasing accuracy for continuous monitoring and classification of these targets.

Therefore, a systematic approach to classify the estimated Doppler features has been presented and confirmed. Moving targets with a continuous variety of velocity have been considered. The results have proven the effectiveness of the tree-structured, selforganizing map (SOM) neural network as a classifier, and Kalman filter as an estimator.

Consequently, accurate information about the targets has been gained. However, a small error will have been occurring in a transient period with the change of the target velocity. Therefore, continuously accurate classification has been gained. Where, as about $99.1 \%$ classification rate was gained and the measurement error is reduced by $95 \%$ for moving targets with a continuous variety of their velocities in noisy environments. Therefore, it can be concluded that using Kalman filter before the proposed classifier makes it a promising candidate for noisy environments.

Therefore, the work presented in this research can be considered as the development of the classification and tracking process for the continuation of the classification process. As well as, this work can be added to the previous works to improve its results better and make them work for the classification with the continuity of change moving targets and not only with trained's

Also, for future work can apply this proposal in the smart car as a smart classifier for continuous automatic monitoring of the surrounding field of the cars.

\section{References}

[1] V. Polfliet, "Processing Radar and Camera Sequences for Person Identification Using a Structured Inference Network", M. Sc. in
Computer Science Engineering, Department of Information Technology, Faculty of Engineering and Architecture, Ghent University, 2018.

[2] J. Hasch, "Driving towards 2020: automotive radar technology trends", In: Proc. of IEEE MTT-S International. Conf. on Microwaves for Intelligent Mobility, pp. 1-4, 2005.

[3] M. Zenaldin, R. M. Narayanan, and R. M. Narayanan, "Radar micro-Doppler based human activity classification for indoor and outdoor environments", In: Proc. of SPIE, Radar Sensor Technology XX, 98291B, Vol. 9829, 2016.

[4] A. Aleksandar, R. Andrew, M. Roderick, and F. Francesco, " Practical classification of different moving targets using automotive radar and deep neural networks", IET Radar, Sonar \& Navigation, Special Issue: Advanced Automotive Sensing - Towards Car Autonomy, Vol. 12, No. 10, pp. 1082-1089, 2018.

[5] J. J. M. de Wit, P. van Dorp, and A. G. Huizing, "Classification of air targets based on rangeDoppler diagrams", In: Proc. of European Radar Conference, pp. 89-92, 2016.

[6] J. P. Guo, J. Xu, L. Yan. X. Xia, X. Xiao, T. Long, and M. Bian, "An improved IMM algorithm based on maneuvering-adaptive model set", In: Proc. of IEEE, International Conference on Radar, pp. 1-5, 2016.

[7] T. T. L. Stanković and I. Djurović, "MicroDoppler-based target detection and feature extraction in indoor and outdoor environments", Adv. Indoor Radar Imaging, Vol. 345, No. 6, pp. 700-722, 2008.

[8] F. Hoffmann, M. Ritchie, F. Fioranelli, A. Charlish, and H. Griffiths, "Micro-Doppler Based Detection and Tracking of UAVs with Multistatic Radar", In: Proc. of IEEE Radar Conference, pp. 1 - 6, 2016.

[9] R. Sadli, C. Tatkeu, K. Hamidoun, Y. El Hillali, and A. Rivenq, "UWB radar recognition system based on HOS and SVMs", IET Radar, Sonar \& Navigation Special Issue: Advanced Automotive Sensing - Towards Car Autonomy, Vol. 12, No. 10, pp. 1137-1145, 2018.

[10] Y. Kim and T. Moon, "Human Detection and Activity Classification Based on MicroDoppler Signatures Using Deep Convolutional Neural Networks", IEEE Geoscience and Remote Sensing Letters, Vol. 13, No. 1, pp. 812, 2016.

[11] L. Yang and G. Li, "Sparsity aware dynamic gesture recognition using radar sensors with angular diversity", IET Radar, Sonar \& 
Navigation, Special Issue: Advanced Automotive Sensing - Towards Car Autonomy, Vol. 12, No. 10, pp. 1114-1120, 2018.

[12] R. E. Kalman, "A new approach to linear filtering and prediction problems", Journal of Basic Engineering, Vol. 82, No. 1, pp. 35-45, 1960.

[13] S. S. Blackman, "Multiple-target tracking with radar applications", Dedham, MA, Artech House, Inc., Vol. 1, p. 463, 1986.

[14] J. J. M. de Wit, P. van Dorp, and A. G. Huizing, "Classification of air targets based on rangeDoppler diagrams", In: Proc. of European Radar Conference, pp. 89-92, 2016.

[15] T. Wagner, R. Feger, and A. Stelzer, "Radar Signal Processing for Jointly Estimating Tracks and Micro-Doppler Signatures", IEEE Access, Vol. 5, pp. 1220 - 1238, 2017.

[16] T. Ye and F. Zhou, "Autonomous space target recognition and tracking approach using star sensors based on a Kalman filter", Applied Optics, Vol. 54, No. 11, 2015.

[17] R. J. Javier and Y. Kim, "Application of linear predictive coding for human activity classification based on micro-Doppler signatures", IEEE Geoscience and Remote Sensing Letters, Vol. 11, No. 10, pp. 1831-1834, 2014.

[18] T. Ichimura and T. Yamaguchi, "A proposal of interactive growing hierarchical SOM", In: Proc. of IEEE International Conference on Systems, Man, and Cybernetics, Anchorage, pp. 3149 - 3154, 2011.

[19] G. Sun, J. Wang, S. Qin, and J. Na, "Radar target recognition based on the multi-resolution analysis theory and neural network", Elsevier Journal Pattern Recognition Letter, Vol. 29, No. 16, pp. 2109-21151, 2008.

[20] T. R. Saeed, M. H. Al-Muifraje, and G. M. Hatem, "Through the Wall, Recognize the Moving Targets Based on Micro-Doppler signatures", International Journal of Electrical and Computer Engineering, Vol. 8, No. 6, 2018.

[21] WWW. Ancortek Inc. 